\title{
Rocket measurements of positive ions during polar mesosphere winter echo conditions
}

\author{
A. Brattli ${ }^{1}$, T. A. Blix ${ }^{1}$, Ø. Lie-Svendsen ${ }^{1}$, U.-P. Hoppe ${ }^{1}$, F.-J. Lübken ${ }^{2}$, M. Rapp ${ }^{2}$, W. Singer ${ }^{2}$, R. Latteck ${ }^{2}$, and \\ M. Friedrich ${ }^{3}$ \\ ${ }^{1}$ Norwegian Defence Research Establishment (FFI), P.O. Box 25, 2027 Kjeller, Norway \\ ${ }^{2}$ Leibniz-Institute of Atmospheric Physics, Kühlungsborn, Germany \\ ${ }^{3}$ Institute of Communication Networks and Satellite Communications, Graz University of Technology, Austria
}

Received: 22 May 2006 - Published in Atmos. Chem. Phys. Discuss.: 26 July 2006

Revised: 25 October 2006 - Accepted: 29 November 2006 - Published: 7 December 2006

\begin{abstract}
On 18 January 2005, two small, instrumented rockets were launched from Andøya Rocket Range $\left(69.3^{\circ} \mathrm{N}\right.$, $\left.16^{\circ} \mathrm{E}\right)$ during conditions with Polar Mesosphere Winter Echoes (PMWE). Each of the rockets was equipped with a Positive Ion Probe (PIP) and a Faraday rotation/differential absorption experiment, and was launched as part of a salvo of meteorological rockets measuring temperature and wind using falling spheres and chaff. Layers of PMWE were detected between 55 and $77 \mathrm{~km}$ by the $53.5 \mathrm{MHz}$ ALWIN radar. The rockets were launched during a solar proton event, and measured extremely high ion densities, of order $10^{10} \mathrm{~m}^{-3}$, in the region where PMWE were observed. The density measurements were analyzed with the wavelet transform technique. At large length scales, $\sim 10^{3} \mathrm{~m}$, the power spectral density can be fitted with a $k^{-3}$ wave number dependence, consistent with saturated gravity waves. Outside the PMWE layers the $k^{-3}$ spectrum extends down to approximately $10^{2} \mathrm{~m}$ where the fluctuations are quickly damped and disappear into the instrumental noise. Inside the PMWE layers the spectrum at smaller length scales is well fitted with a $k^{-5 / 3}$ dependence over two decades of scales. The PMWE are therefore clearly indicative of turbulence, and the data are consistent with the turbulent dissipation of breaking gravity waves. We estimate a lower limit for the turbulent energy dissipation rate of about $10^{-2} \mathrm{~W} / \mathrm{kg}$ in the upper $(72 \mathrm{~km})$ layer.
\end{abstract}

\section{Introduction}

Polar mesosphere winter echoes (PMWE) are layers of enhanced radar echoes occuring between about 55 and $85 \mathrm{~km}$ altitude in the high-latitude mesosphere during winter, with an occurence rate of $2.9 \%$ (Zeller et al., 2006). They are

Correspondence to: A. Brattli

(alvin.brattli@ffi.no) less intense, occur more sporadically, and occur at lower altitudes than their summer counterpart, polar mesosphere summer echoes (PMSE), which occur in the mesopause region, typically between 80 and $90 \mathrm{~km}$. Both phenomena require fluctuations in the electron density on a scale of at most a few meters to be detected by a $50-\mathrm{MHz}$ radar, although the magnitude of the fluctuations needs to be much larger for PMSE than for PMWE, to account for the much stronger PMSE radar backscattered signal. PMWE are primarily seen at daytime (Kirkwood et al., 2002; Zeller et al., 2006), and their occurrence is strongly correlated with solar proton events or enhanced high-energy electron precipitation, which indicates that large electron densities are a necessary prerequisite for their occurrence.

PMSE are by now fairly well understood, being caused by the presence of large (a few nanometers or more) particles, probably created by condensation of supersaturated water vapour at the extremely cold summer mesopause. Such large particles quickly become electrically charged by the plasma. In order to preserve charge neutrality in the plasma, free electrons are essentially "frozen" to the ice particles, with density fluctuations that mirror the ice particle density fluctuations. Once small-scale ice-particle density fluctuations have been created, for instance through turbulence, their low diffusivity then implies that the corresponding small-scale electron density fluctuations may last for a long time, up to hours, even in the absence of active turbulence. The time constant for destruction of fluctuations depends on particle size $\left(r^{2}\right)$, i.e. on altitude (Lie-Svendsen et al., 2003; Rapp and Lübken, 2004).

PMWE are currently not well understood, despite that the first observations of mesospheric radar echoes were made in 1978 at mid latitudes with the SOUSY VHF radar (Czechowsky et al., 1979) and at high latitudes in 1979 by the Poker Flat radar in Alaska (Ecklund and Balsley, 1981; Balsley et al., 1983). The polar winter mesosphere is much too warm for water ice particles to form, and therefore the

Published by Copernicus GmbH on behalf of the European Geosciences Union. 
mechanism explaining PMSE cannot explain PMWE. In the absence of heavy, charged particles to influence the electron motion, meter-scale density fluctuations should be strongly damped, even in the presence of active turbulence, because viscous dissipation of turbulent eddies becomes important at these length scales. Without invoking very large energy dissipation rates or without large background electron densities and gradients, it has been speculated that the fluctuations would be much too small to explain the radar echoes (Belova et al., 2005). Kirkwood et al. (2002) have therefore proposed that charged aerosols may cause PMWE after all, corroborated by lidar measurements of enhanced backscatter that Stebel et al. (2004) interpreted as aerosol layers. Since ice particles are ruled out, Stebel et al. suggested that ablation and recondensation of meteoric material could be a source of aerosol particles. This meteoric smoke (Hunten et al., 1980) has also been suggested as the condensation nuclei on which PMSE ice particles grow, and recent in situ measurements have shown that charged nano-particles do exist in the winter mesosphere (Lynch et al., 2005; Rapp et al., 2005). However, the lidar observations would require roughly a factor $10^{3}$ more dust particles than modelled by Hunten et al., or a large increase in dust particle size. Until such particles have been better characterised, it remains an open question whether they can explain PMWE.

To resolve these issues, radar measurements are not sufficient, as these only contain information about fluctuations in the electron density. The required detailed information about the state of the mesosphere during PMWE must be obtained in situ by sounding rockets. Recently, Lübken et al. (2006) presented results from a series of meteorological and instrumented rockets launched into PMWE from Andøya Rocket Range in January 2005, where it was shown that even weak turbulence can create PMWE, without involving charged aerosol particles.

This paper is an extension of the study by Lübken et al. (2006), analysing the data from the instrumented rockets in considerably more detail. Although electron densities from the PMWE scattering regions, approximately $65-70 \mathrm{~km}$, are not directly available from these rocket flights, measurements around $60 \mathrm{~km}$ by the Faraday rotation technique found electron densities up to $n_{e} \approx 10^{10} \mathrm{~m}^{-3}$, which are extremely large densities for this altitude region, and even higher than measured in the auroral zone at these heights. "Pragmatically extending" these densities into the PMWE scattering region, Lübken et al. found that the radar echoes could in fact be explained by turbulence without invoking unrealistically high energy deposition rates. In other words, in these particular cases PMWE could be explained from "normal" turbulence alone, without invoking aerosol particles to reduce the electron diffusivity.

In this paper, we analyze the positive ion density measurements using the wavelet transform technique. This technique allows us to extract the altitude dependence of the small-scale plasma density fluctuations, and easily identify the regions of most interest for the generation of PMWE. Section 2 gives a short summary of the wavelet technique that we use. Section 3 gives a brief presentation of the rocket ion probe, and how the measured current is converted into density. Section 4 presents the main results of the wavelet analysis, while Section 5 discusses implications for energy deposition rates.

\section{Wavelets}

The Wavelet Transform (WT) is in many ways superior to the traditional Windowed Fourier Transform (WFT), overcoming many of its time-frequency localization problems. Among the advantages of the WT over the WFT is that it has good time resolution for high-frequency signals and good frequency resolution for low-frequency signals, whereas with the WFT, you can get good time resolution (with a narrow window) or good frequency resolution (with a wide window), but not both simultaneously. For the WFT, the time/frequency resolution is related through $\sigma_{t} \sigma_{\omega}=$ const. $\geq 1 / 2$, where $\sigma_{t}$ and $\sigma_{\omega}$ are the time and frequency widths, related to the shape of the window function. The product $\sigma_{t} \sigma_{\omega}$ is smallest for Gaussian window functions. For the WT, the time and frequency resolutions are expressed as $s \sigma_{t}$ and $\sigma_{\omega} / s$ (and thus depend on the scale, $s$, defined below), although $\sigma_{t} \sigma_{\omega}$ still remains constant at all scales (Mallat, 1999). This is analogous to the Heisenberg uncertainty principle. Due to these advantages of the WT over the WFT, Strelnikov et al. (2003) recently applied this technique to the analysis of neutral air turbulence in the mesosphere, and is also the reason we have chosen to use wavelets in this analysis.

The WT of the discrete time series $x_{n}$ is given by the convolution

$W_{n}(s)=\sum_{n^{\prime}=0}^{N-1} x_{n^{\prime}} \Psi^{*}\left(\frac{\left(n^{\prime}-n\right) \delta t}{s}\right)$

where

$\Psi\left(\frac{\left(n^{\prime}-n\right) \delta t}{s}\right)=\left(\frac{\delta t}{s}\right)^{1 / 2} \Psi_{0}\left(\frac{\left(n^{\prime}-n\right) \delta t}{s}\right)$

is the scaled, translated, and normalised (to unit energy) version of the "mother wavelet" $\Psi_{0}(\eta)$, and the * denotes the complex conjugate. The sampling time interval is $\delta t$, and the wavelet scales $s$ are given by

$s_{j}=s_{0} 2^{j \delta j}, j=0,1, \ldots, \frac{1}{\delta j} \log _{2}\left(\frac{N \delta t}{s_{0}}\right)$

where $\delta j$ determines the sampling in scale (smaller $\delta j$ gives a better resolution). The smallest scale $s_{0}$ is given by $2 \delta t$. In the present work, we have used the usual approximation to the Morlet wavelet (Foufoula-Georgiou and Kumar, 1994),

$\Psi_{0}(\eta)=\pi^{-1 / 4} e^{i \Omega_{0} \eta} e^{-\eta^{2} / 2}$ 
where $\Omega_{0}$ is a nondimensional frequency. For the Morlet wavelet, the equivalent Fourier period is given by

$T_{F}(s)=\frac{4 \pi s}{\Omega_{0}+\sqrt{2+\Omega_{0}^{2}}}$.

For numerical computations, it is considerably faster to compute the WT in Fourier space:

$W_{n}(s)=\sum_{k=0}^{N-1} \hat{x}_{k} \hat{\Psi}^{*}\left(s \omega_{k}\right) e^{i \omega_{k} n \delta t}$

where

$\omega_{k}= \begin{cases}\frac{2 \pi k}{N \delta t} & \text { if } k \leq \frac{N}{2} \\ -\frac{2 \pi(N-k)}{N \delta t} & \text { if } k>\frac{N}{2}\end{cases}$

and the Fourier transformed quantities $\hat{x}_{k}$ and $\hat{\Psi}$ are given by

$\hat{x}_{k}=\frac{1}{N} \sum_{n=0}^{N-1} x_{n} e^{-2 \pi i k n / N}$

and

$\hat{\Psi}\left(s \omega_{k}\right)=\left(\frac{2 \pi s}{\delta t}\right)^{1 / 2} \hat{\Psi}_{0}\left(s \omega_{k}\right)$

respectively, where

$\hat{\Psi}_{0}(s \omega)=\pi^{-1 / 4} H(\omega) e^{-\left(s \omega-\Omega_{0}\right)^{2} / 2}$

for the Morlet wavelet, and

$H(\omega)= \begin{cases}0 & \text { if } \omega \leq 0 \\ 1 & \text { if } \omega>0\end{cases}$

is the Heaviside step function. Note that $\hat{\Psi}_{0}$ is normalised to have unit energy, i.e. that $\int_{-\infty}^{+\infty}\left|\hat{\Psi}_{0}(\Omega)\right|^{2} d \Omega=1$. For the present analysis, $\Omega_{0}=48$, and $\delta j=0.0125$. These values were chosen in order to properly resolve the spin frequency and its harmonics.

The wavelet analysis was performed on the time series of the relative ion density fluctuations,

$n_{i}=\frac{N_{i}-\left\langle N_{i}\right\rangle}{\left\langle N_{i}\right\rangle}$

where $N_{i}$ is the calculated ion density, and $\left\langle N_{i}\right\rangle$ is the running mean of $N_{i}$ over 50 spin periods.

We will not go into more details on the theory of wavelets here, but refer to some of the extensive literature available for details, e.g. Foufoula-Georgiou and Kumar (1994), Torrence and Compo (1998), Mallat (1999), and references therein.

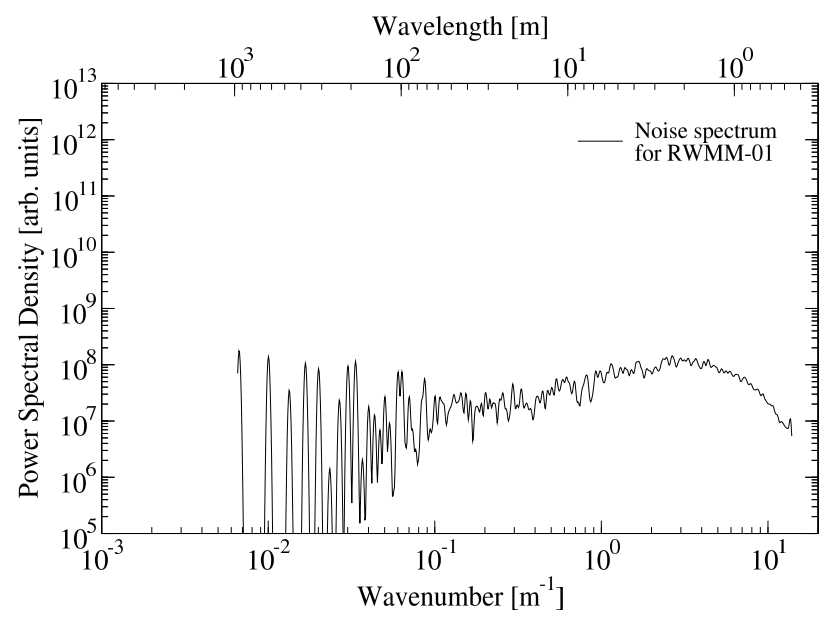

Fig. 1. Wavelet power spectral density for the RWMM-01 PIP directly before launch, while the payload was at the launch pad. For the wavelength, a velocity of $560 \mathrm{~m} / \mathrm{s}$ is assumed, which corresponds to an altitude of $62.6 \mathrm{~km}$ for this payload.

\section{Instrumentation and measurements}

The two instrumented payloads in the rocket salvo were identical, and they carried instruments for measuring number densities of positive ions and electrons. Positive ion density was measured using a Positive Ion Probe (PIP) (Blix et al., 1990b; Sagalyn et al., 1963) in the front of the payload. These PIPs consisted of an outer grid (25 mm diameter) at payload potential (floating potential) and an inner electrode biased at $-6 \mathrm{~V}$ relative to the outer grid, thus attracting positive ions. The inner electrode was connected to an electrometer with 4 decades automatic ranging, and a 12bit linear digital to analog converter with a sampling rate of $2441.4 \mathrm{~Hz}$, giving a theoretical resolution of $\sim 0.02 \%$ for the fluctuations, and a spatial resolution of better than $\sim 0.5 \mathrm{~m}$. However, noise in the electronics raised the noise floor, so the actual spatial resolution was around 5-7 $\mathrm{m}$ inside the PMWE layers, where the small-scale signal was strongest. Fig. 1 shows the wavelet power spectral density of this noise from the PIP of RWMM-01 directly before launch, while the rocket was still at the launch pad. The noise spectrum has a characteristic shape, with a broad peak in the higher frequencies. The shape of the noise spectrum does not change significantly for the entire flight (for both flights), thus making it easy to identify in the wavelet spectra.

Absolute electron density measurements were made with a Faraday rotation/differential absorption experiment that was also included on the payloads. These measurements have a height resolution on the order of $1 \mathrm{~km}$, as the payloads must complete a full spin revolution to get one data point. Linearly polarised RF waves are transmitted from the ground to the flying payload and the receiving antenna, spinning with the payload, scans the polarisation at the altitude under inves- 


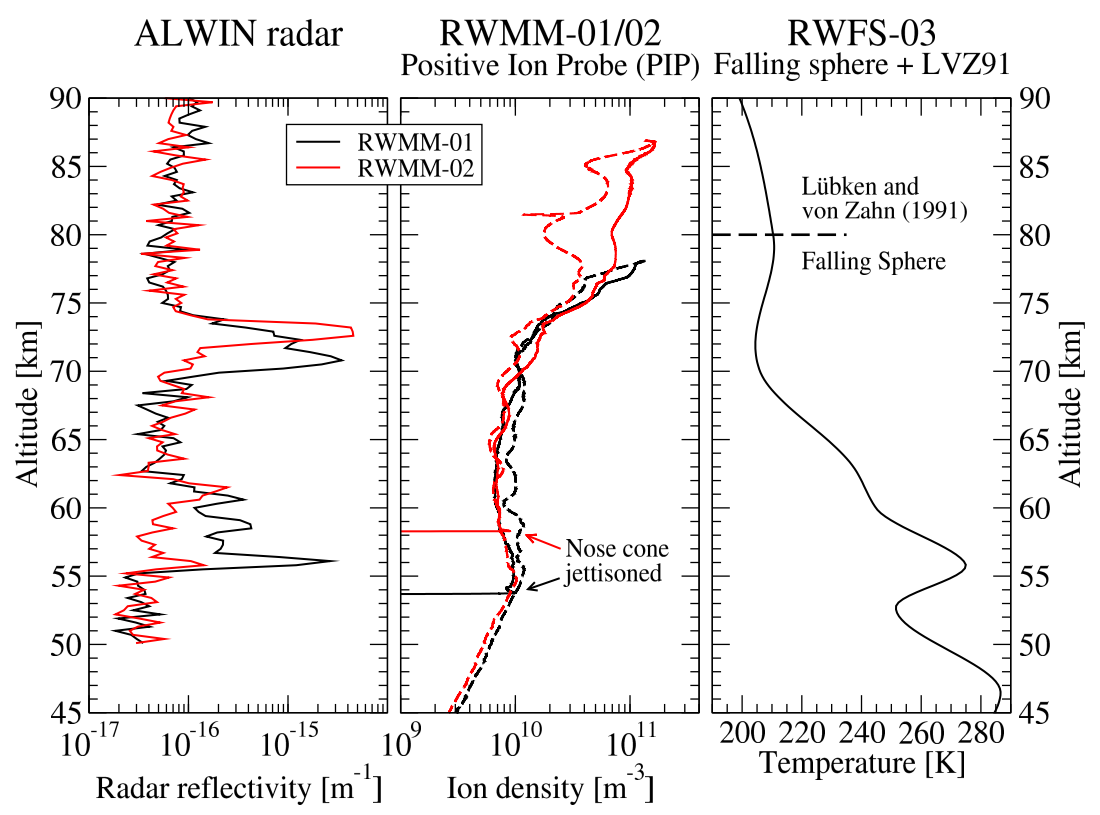

Fig. 2. Left panel: PMWE, as measured by the ALWIN radar. Integration times for the two radar profiles were $\sim 18.5$ min, centered around the launch times of the rockets. Middle panel: Ion densities as calculated from the PIP measurements on the instrumented payloads, averaged over one spin cycle. The red and black curves denote RWMM-01 and RWMM-02, respectively, while solid and dashed lines denote up- and downleg. The nosecone was jettisoned at $53.7 \mathrm{~km}(58.3 \mathrm{~km})$, and there are thus no data below this on upleg. Right panel: Vertical temperature profile as measured by the first falling sphere launched after the instrumented payloads. Above the dashed line we have used the temperature profile for January from Lübken and von Zahn (1991).

Table 1. Launch times and trajectory data for the instrumented payloads. Launch date was 18 January 2005.

\begin{tabular}{lcc}
\hline & RWMM-01 & RWMM-02 \\
\hline Launch time (UTC) & $11: 36: 30$ & $12: 06: 00$ \\
Nose cone jettisoned & $53.7 \mathrm{~km}$ & $58.3 \mathrm{~km}$ \\
Apogee & $78 \mathrm{~km}$ & $86.9 \mathrm{~km}$ \\
Spin frequency & $13.7 \mathrm{~Hz}$ & $13.3 \mathrm{~Hz}$ \\
\hline
\end{tabular}

tigation. The rate of change of the polarisation (orientiation, ellipticity) is a function of the electron density. This type of measurement is insensitive to distortions caused by payload charging or aerodynamic effects (Mechtly, 1974; Jacobsen and Friedrich, 1979).

The payloads were launched on 18 January 2005 from Andøya Rocket Range $\left(69.3^{\circ} \mathrm{N}, 16^{\circ} \mathrm{E}\right)$ during a period with solar radiation storms and increased geomagnetic activity. Around this time the GOES-11 satellite showed proton fluxes that were about a factor $10^{3}$ larger than during quiet conditions. Launch times and trajectory data for the instrumented payloads (RWMM-01 and RWMM-02) are listed in Table 1. The distance between the ALWIN radar measurements and the rockets were $\approx 7.8 \mathrm{~km}(\approx 25 \mathrm{~km})$ at $56 \mathrm{~km}$ altitude on upleg (downleg) for RWMM-01, and $\approx 5.8 \mathrm{~km}(\approx 22 \mathrm{~km})$ for RWMM-02.
Conversion from ion current to ion density was done using (Sagalyn et al., 1963)

$$
I_{i}=\pi r^{2} N_{i} q_{e} v_{i} f(V) \cdot\left[\frac{1}{2} e^{-x^{2}}+\sqrt{\pi}\left(\frac{x}{2}+\frac{1}{4 x}\right) \operatorname{erf}(x)\right],
$$

where $I_{i}$ is the ion current, $x=2 v_{r} / \sqrt{\pi} v_{i}, r$ is the radius of the outer grid of the PIP $(0.0125 \mathrm{~m}), N_{i}$ is the ion density, $q_{e}$ is the elementary charge, $v_{i}$ is the ion thermal velocity, $v_{r}$ is the payload velocity, and

$\operatorname{erf}(x)=\frac{2}{\sqrt{\pi}} \int_{0}^{x} e^{-\xi^{2}} d \xi$

is the error function. The function $f(V)$ is a function of the payload potential (floating potential) $V$, and requires a special probe mode and analysis (Sagalyn et al., 1963). This mode was not available on these instruments, so $f(V)$ is set to unity (Folkestad, 1970). The error introduced by this uncertainty should not be large: The mesospheric conditions during the rocket flights will cause a negative payload potential, in which case $f(V)$ is only weakly dependent on $V$ (the ions are attracted by the payload). Moreover, we shall focus on relative fluctuations in ion density on scales smaller than $\sim 10^{3} \mathrm{~m}$, which are not influenced by slow changes in the payload potential. Finally, setting $f(V)=1$ for RWMM01 yields a positive ion density at $60 \mathrm{~km}$ that agrees within a factor two with the electron density obtained from the wave propagation experiment. 

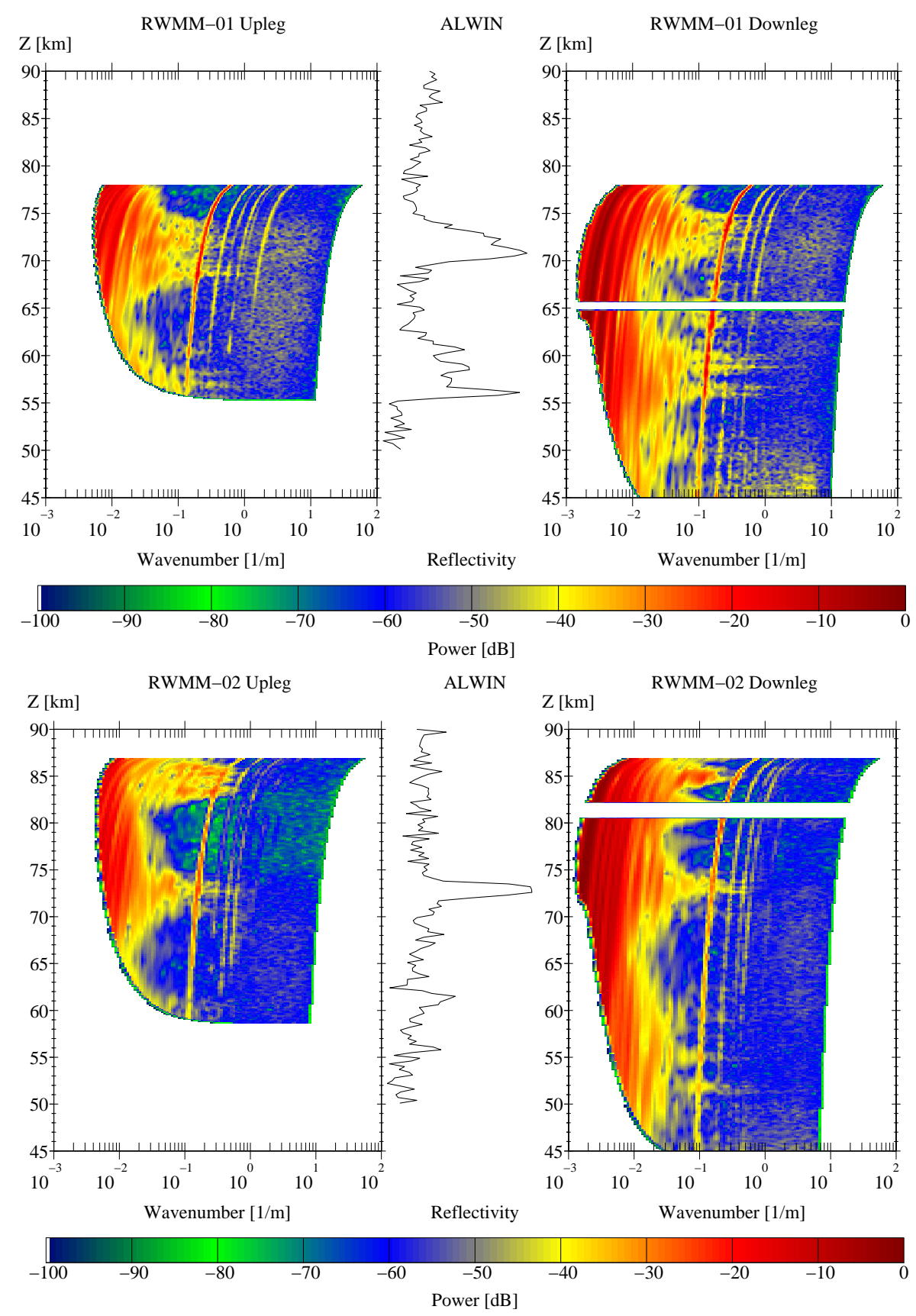

Fig. 3. Wavelet transform of relative fluctuations in ion density for RWMM-01 (top) and RWMM-02 (bottom). The wavelet transform for upleg (downleg) is at the left (right), while an ALWIN radar echo profile taken near the time of launch is shown in the middle. The payload spin frequency and its harmonics are clearly visible throughout the entire flight for both payloads. The horizontal white patches indicate masking of the data due to data dropout.

\section{Results}

The mesospheric conditions at the time of the rocket launches are shown in Fig. 2. The ion densities, as calculated by solving Eq. (12) for $N_{i}$, are shown together with PMWE radar profiles and the neutral temperature as measured with the first falling sphere launched after the instrumented rockets. At the top of the trajectory for the falling sphere the temperature data are unreliable, due to a rather long reaction time of the sphere to density changes in the atmosphere, so we have used the average January temperature profile from Lübken and von Zahn (1991) above $80 \mathrm{~km}$, with a spline 

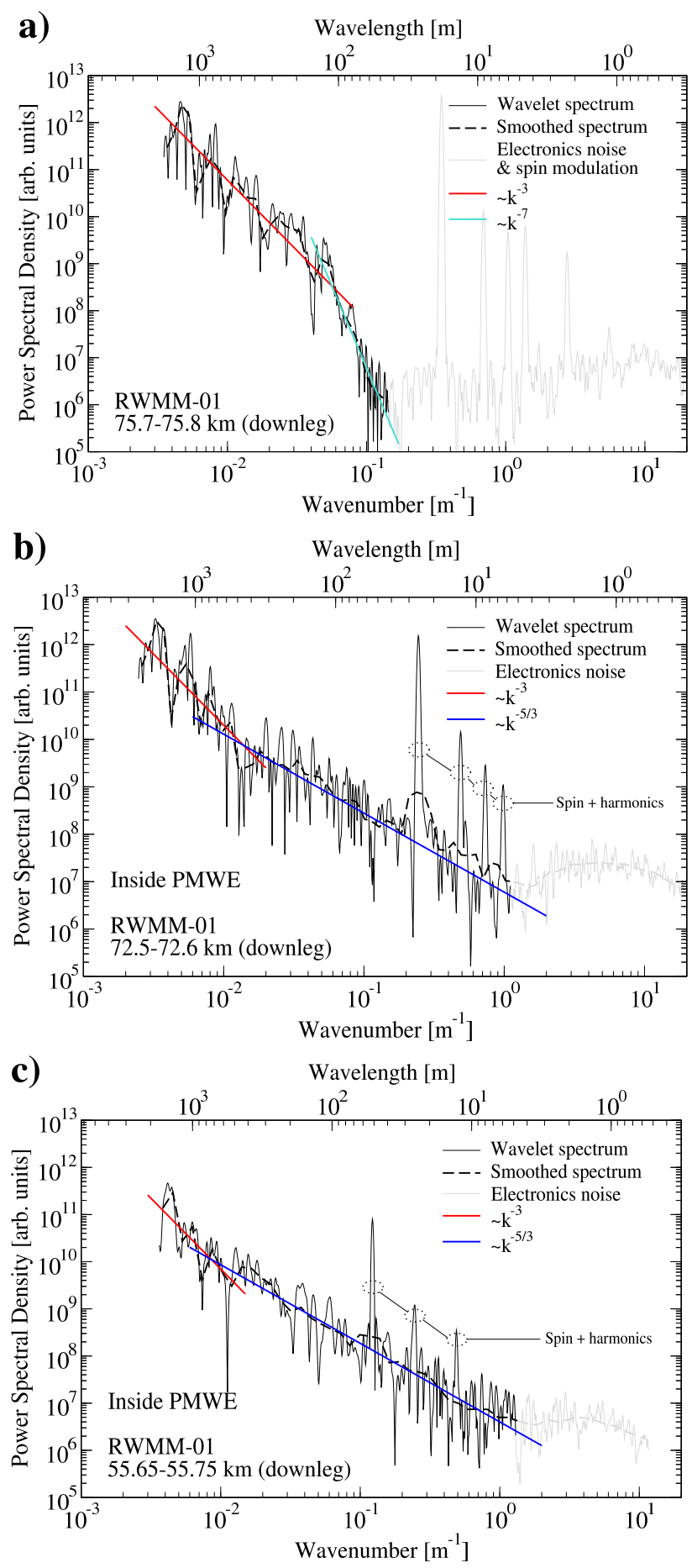

Fig. 4. Positive ion spectra for RWMM-01 at selected altitudes a) outside and b,c) inside PMWE altitude ranges. The gray parts of the spectra indicate the instrumental noise level. The $k^{-5 / 3}, k^{-3}$, and $k^{-7}$ parts of the spectra are indicated with red, blue, and light blue lines, respectively. These lines are drawn to guide the eye, they have not been fitted. interpolation to merge the two profiles. The ion density profiles shown in Fig. 2 have been averaged over one payload spin cycle to make the graph less cluttered.

The ion densities in Fig. 2 are about two orders of magnitude higher than what is normal for the season (Bilitza, 2001; Lübken et al., 2006), caused by the high solar activity at the time of the rocket campaign.

In Fig. 2 we have also plotted the radar echoes, as measured by the ALWIN radar at the time of launch for each rocket. The PMWE measured at the time of the launch of the first payload consisted of two layers $(\sim 55-63 \mathrm{~km}$ and $\sim 70$ $75 \mathrm{~km}$ ). The lowest layer subsequently split up into two layers, and eventually died out. By the time the second payload was launched, the remains of the lowest layer were just above the detection threshold of the radar. Thus, this layer was still there, but only barely visible.

The wavelet transforms of the positive ion data from the two rocket flights are shown in Fig. 3, with spectral power density as a function of wavenumber and altitude. Portions of the data have been masked out due to data dropout and other erroneous behaviour, and can be seen as horizontal, white stripes on downleg on both flights. Boundary effects from the start and the end of the time series (the cone of influence; COI) have also been masked out, and the result can be seen as a gradual limitation of low wavenumbers in the lower part of each leg. The instantaneous payload velocity was used for calculating the wavenumber from the period $T_{F}(s)$ at each altitude. The spin frequencies $(13.7 \mathrm{~Hz}$ for RWMM-01 and $13.3 \mathrm{~Hz}$ for RWMM-02) can be extracted from the raw data with high accuracy, and are clearly visible along with their harmonics in Fig. 3 as thin bands with high power density. The spin frequency itself is the most dominating of these, but the harmonics are also clearly visible (increasing frequency towards the right). Since the payload velocities decrease with increasing altitude, we have higher spatial resolution near apogee than at lower altitudes. From Fig. 3 we can clearly see enhancements in the power spectral density at precisely those altitude ranges where PMWE were measured (55-63 km and 68-75 km for RWMM-01; 55-63 km and 70-75 km for RWMM-02).

In the altitude ranges with PMWE, we have more power at high wavenumbers (short wavelenghts) compared with where we do not have PMWE, and the enhanced fluctuations are confined to several narrow subranges. While turbulence is clearly involved, as we will show below, the mechanism behind these narrow subranges is not known, but a likely candidate is breaking gravity waves.

For RWMM-01, the upper ledge of the topmost layer is located at $74.5 \mathrm{~km}$ on upleg, and $75.1 \mathrm{~km}$ on downleg. The horizontal distance travelled by the payload between the crossings of the layer ledges is $6.75 \mathrm{~km}$. Neglecting drift of the layer and assuming that the situation did not change significantly during the $50 \mathrm{~s}$ between the crossings, we get a tilt of the layer of approximately $5^{\circ}$. For RWMM-02, the upper ledge is located at $74.35 \mathrm{~km}$ on upleg and $74.7 \mathrm{~km}$ on 
a)

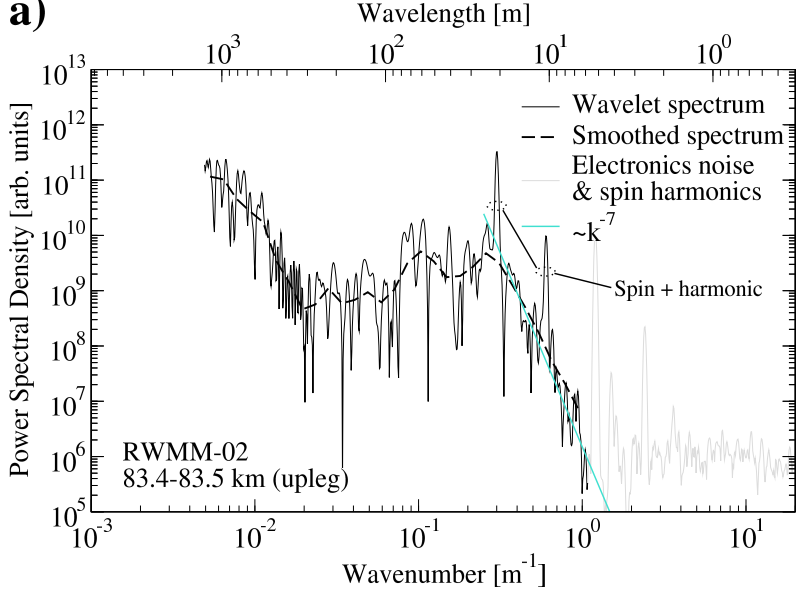

c)

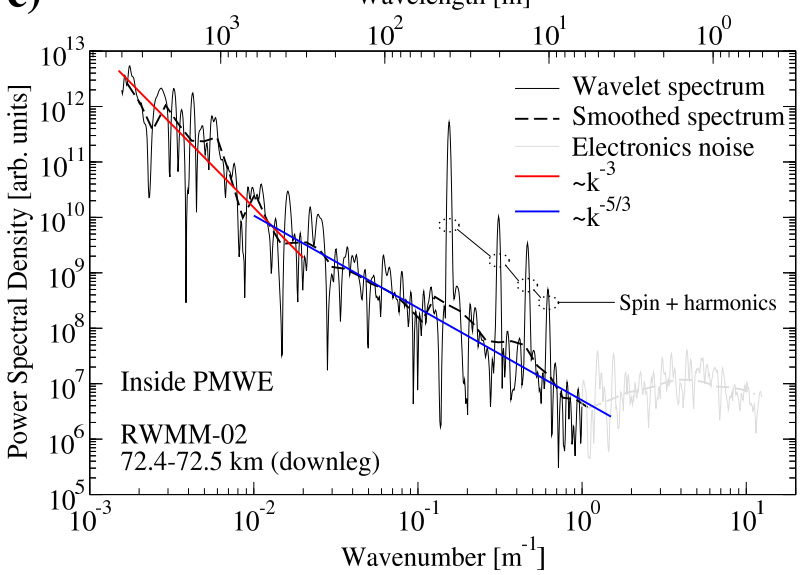

b)

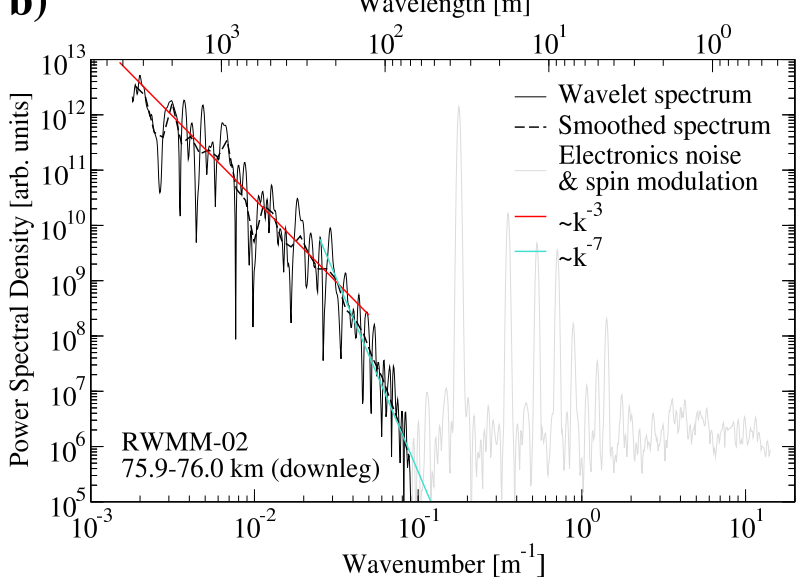

d)

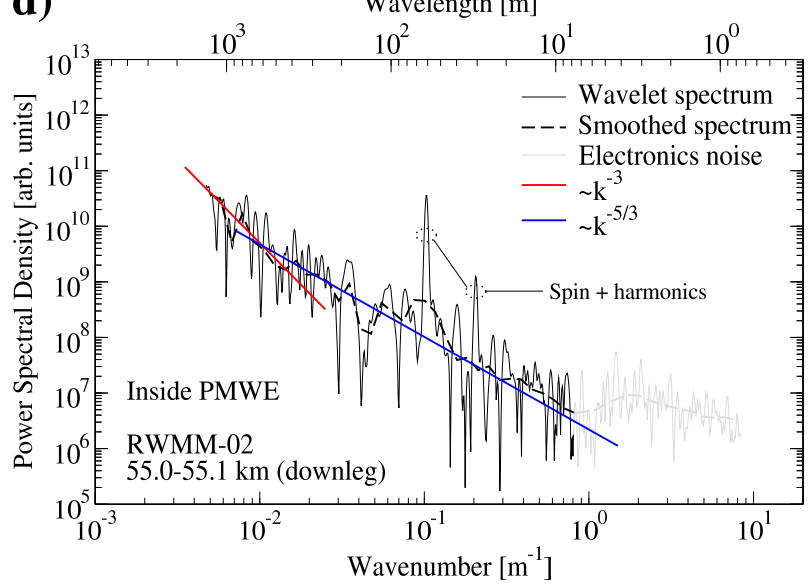

Fig. 5. Positive ion spectra for RWMM- 02 at selected altitudes a) outside PMWE, but inside the assumed plasma instability, b) outside PMWE, and c,d) inside PMWE altitude ranges. The gray parts of the spectra indicate the instrumental noise level. The $k^{-5 / 3}, k^{-3}$, and $k^{-7}$ parts of the spectra are indicated with red, blue, and light blue lines, respectively. These lines are drawn to guide the eye, they have not been fitted.

downleg (100 s time difference). The horizontal separation between the ledge crossings was $10.9 \mathrm{~km}$, giving a tilt of about $1.8^{\circ}$.

The heavy influence of the payload spin frequency and its harmonics right in the middle of the interesting frequency range of the time series makes it difficult to remove the spin from the time series, without also filtering out a lot of data. This, in combination with the dominating electronics noise at high frequencies (see Fig. 1) makes estimating the magnitude of the ion density fluctuations problematic. Still, the magnitude of the fluctuations on scales larger than one spin period can be estimated by

$\Delta n_{i}=\frac{\left\langle N_{i}\right\rangle_{1}-\left\langle N_{i}\right\rangle_{50}}{\left\langle N_{i}\right\rangle_{50}}$

where $\langle\cdot\rangle_{m}$ is a running average over $m$ spin periods. The above estimate gives us ion density fluctuations on the order of $1-3 \%$ for lengths between one and 50 spin periods along the rocket trajectory. Fluctuations due to spin can be as large as $10 \%$.

In Figs. 4 and 5 we compare typical ion density fluctuation spectra from outside and inside the PMWE layers. The ion density fluctuation spectra within the PMWE show characteristics that we would expect from turbulence, with the slope being almost proportional to $k^{-5 / 3}$, where $k=2 \pi / \lambda$ and $\lambda$ is the wavelength, over a significant part of the frequency range, indicating the inertial subrange of a turbulent cascade. The $k^{-5 / 3}$ slope extends from $k \sim 10^{-2} \mathrm{~m}^{-1}$ (corresponding to wavelengths of roughly $400-600 \mathrm{~m}$ ) and up to $k \sim 1 \mathrm{~m}^{-1}$ (wavelengths of roughly 6-10 m), where the signal drops below the electronics noise. In these ranges, a least squares fit (after removing spin and its harmonics) gives us slopes ranging from $-5 / 3-0.19$ to $-5 / 3+0.14$. The noise originating in the onboard electronics prevented us from measuring fluctuations all the way down to the Bragg wavelength $(2.8 \mathrm{~m})$ of the ALWIN radar (see the light gray parts of the curves in Figs. 4 and 5). 


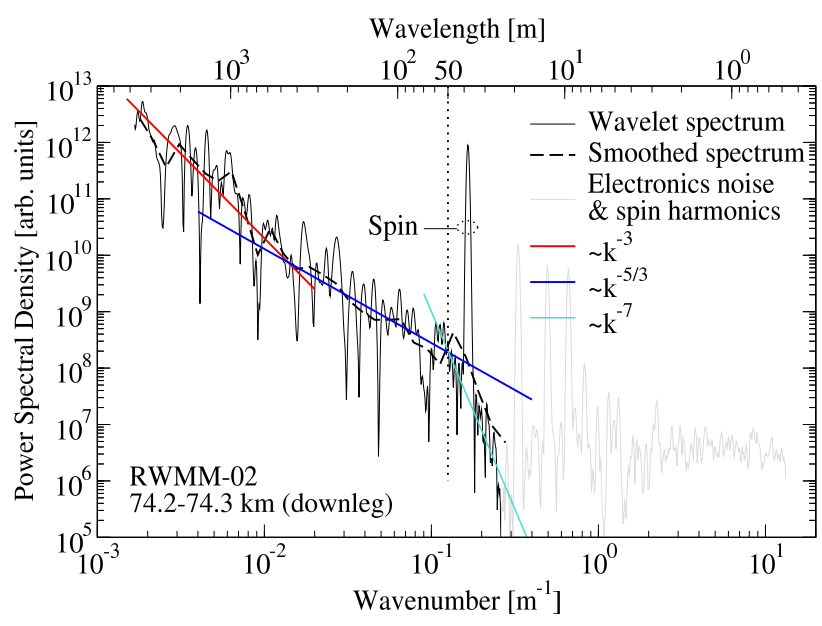

Fig. 6. An example of a transitional spectrum where we can see slopes proportional to $k^{-3}, k^{-5 / 3}$, and $k^{-7}$. The inner scale is at approximately $50 \mathrm{~m}$.

For $k \lesssim 10^{-2} \mathrm{~m}^{-1}$ the spectra are very close to being proportional to $k^{-3}$, which indicates saturated gravity waves (Blix et al., 1990a,b). Our interpretation of these data is that we have gravity waves breaking in the altitude ranges $\approx 55-$ 60 and $\approx 70-75 \mathrm{~km}$, giving rise to turbulence that dissipates its energy at scales $\lesssim 10 \mathrm{~m}$.

In the altitude ranges where we do not see PMWE there are no signs of a turbulent cascade with a $k^{-5 / 3}$ power spectrum. Instead we see buoyancy range spectra with a $k^{-3}$ slope up to $k \approx 4 \cdot 10^{-2} \mathrm{~m}^{-1}$ (wavelengths down to about $150 \mathrm{~m}$ ), at which point the slope of the spectrum changes directly to $\sim k^{-7}$. This indicates that we have gravity waves that do not create turbulence. Alternatively, the energy dissipation rate is so low (see below) that there is not "room" for an inertial subrange, so that the wave energy flux is converted directly to viscous heating without going through a turbulent cascade.

In the uppermost part of the RWMM-02 flight (above $82 \mathrm{~km}$ ), strong ion density fluctuations were seen, while no PMWE echoes were seen in this region. This is consistent with these ion fluctuations being strongly damped before they reach the $k \sim 1 \mathrm{~m}^{-1}$ region where PMWE are generated. These fluctuations therefore seem to be unrelated to the PMWE echoes. An ion density fluctuation spectrum from this region is shown in Fig. 5a. One possibility is that they are generated by the two-stream instability described by Dimant and Sudan (1995). Observational evidence for this type of instability has been presented by Blix et al. (1996). Usually, this type of plasma instability is restricted to heights above $90 \mathrm{~km}$, but may, under favourable conditions, be located even below $80 \mathrm{~km}$. This instability requires the presence of an electric field (usually $>50 \mathrm{mV} / \mathrm{m}$ ). Since electric fields were not measured, we may only hypothesize that this is the cause. We will not discuss this further in this paper.

\section{Energy dissipation rates}

If neutral air turbulence alone were responsible for stirring up the ion density fluctuations (and hence also the electron density fluctuations) needed to get PMWE, the turbulence must have created density fluctuations all the way down to the Bragg wavelength of the radar. The smaller the scale where we still have a turbulent cascade, the higher the energy dissipation rate will be. The inner scale, $\ell_{0}=2 \pi / k_{0}$, where the asymptotic forms of the spectra in the inertial $\left(k^{-5 / 3}\right)$ and viscous $\left(k^{-7}\right)$ subranges merge, is related to the energy dissipation rate $\epsilon$ through (Lübken et al., 1993)

$\ell_{0}=9.9\left(\frac{v^{3}}{\epsilon}\right)^{1 / 4}$,

where $v$ is the kinematic viscosity. Since the electronics noise prevents us from identifying the transition between the inertial and viscous subranges (the inner scale) in the turbulent spectra of Figs. 4 and 5, we cannot calculate $\epsilon$ using a spectral fit. We can therefore only calculate a lower limit for $\epsilon$. If we let $l_{N}$ be the wavelength where the $k^{-5 / 3}$ spectrum disappears into the noise, the inner scale must be smaller than this, $\ell_{0}<l_{N}$. Hence the lower limit on the energy dissipation rate is given by

$\epsilon>v^{3}\left(\frac{9.9}{l_{N}}\right)^{4}$.

From Figs. 4 and $5 l_{N} \approx 10 \mathrm{~m}$. Thus, the inner scale $\ell_{0}$ must be smaller than this for both PMWE layers.

Table 2 lists the corresponding limits on the energy dissipation rate. We note that the lower limit on $\epsilon$ is very small for the lowest PMWE layer, and on the order of $10^{-2} \mathrm{~W} / \mathrm{kg}$ for the upper layer. From Eq. (14) $\epsilon$ is very sensitive to the value of $\ell_{0}, \epsilon \propto \ell_{0}^{-4}$. This is also illustrated in Table 2 , showing that if the inner scale is on the order of the radar wavelength, $\ell_{0}=\lambda_{0}$, the required energy dissipation rate increases by an order of magnitude for both layers. And if the inner scale matches the Bragg wavelength, $\ell_{0}=\lambda_{0} / 2$, the energy dissipation rate becomes very high for the upper layer $(\epsilon \approx 2 \mathrm{~W} / \mathrm{kg})$, while it is still in the $\mathrm{mW} / \mathrm{kg}$ range for the lower layer. A heating rate of more than $10^{-2} \mathrm{~W} / \mathrm{kg}$ (about $1 \mathrm{~K} /$ day) for the upper layer is more than a factor 10 larger than the typical winter time measurements by Lübken et al. (1993).

The transition between turbulent and non-turbulent spectra happens over just a few hundred meters, at the top and bottom of the PMWE layers, and the spatial distribution of turbulent/non-turbulent spectra matches the height variation of PMWE/no PMWE exactly. Fig. 6 shows an example of a "transitional" spectrum from directly above the topmost PMWE layer for RWMM-02. We note that the power spectral density at small wavenumbers is quite similar to the turbulent spectra, both in slope and magnitude, but it quite suddenly drops off at $k \approx 10^{-1} \mathrm{~m}^{-1}$. Further up (away from the PMWE layer), the power spectral density drops off at smaller 
Table 2. Calculated energy dissipation rate $\epsilon$ inside PMWE, for the observed case where the inner scale $\ell_{0}$ is smaller than $l_{N} \approx 10 \mathrm{~m}$, and for the hypothetical cases where $\ell_{0}=\lambda_{0}$, and $\ell_{0}=\lambda_{0} / 2$, where $\lambda_{0}=5.6 \mathrm{~m}$ is the radar wavelength. The kinematic viscosity, $v$, is taken from the U.S. Standard Atmosphere, 1976.

\begin{tabular}{llrrrr}
\hline & \multicolumn{2}{c}{ RWMM-01 } & \multicolumn{2}{c}{ RWMM-02 } \\
\hline Altitude & {$[\mathrm{km}]$} & $55.65-55.75$ & $72.5-72.6$ & $55.0-55.1$ & $72.4-72.5$ \\
$v$ & {$\left[\mathrm{~m}^{2} / \mathrm{s}\right]$} & $3.2 \cdot 10^{-2}$ & 0.24 & $2.9 \cdot 10^{-2}$ & 0.24 \\
$\epsilon\left(\ell_{0}<l_{N}\right)$ & {$[\mathrm{W} / \mathrm{kg}]$} & $>3.1 \cdot 10^{-5}$ & $>1.4 \cdot 10^{-2}$ & $>2.5 \cdot 10^{-5}$ & $>1.3 \cdot 10^{-2}$ \\
$\epsilon\left(\ell_{0}=\lambda_{0}\right)$ & {$[\mathrm{W} / \mathrm{kg}]$} & $3.0 \cdot 10^{-4}$ & 0.14 & $2.5 \cdot 10^{-4}$ & 0.14 \\
$\epsilon\left(\ell_{0}=\lambda_{0} / 2\right)$ & {$[\mathrm{W} / \mathrm{kg}]$} & $4.9 \cdot 10^{-3}$ & 2.3 & $3.9 \cdot 10^{-3}$ & 2.2 \\
\hline
\end{tabular}

$k$, while further down (closer to the PMWE layer), it happens at a larger $k$. If we still interpret the spectrum as a turbulent spectrum, hence identifying the change in slope as the location of the inner scale, the increase in inner scale compared to the spectra inside PMWE (where we could only put an upper limit on the inner scale) indicates that the energy dissipation is much lower than in the turbulent layer. With $\ell_{0} \approx 50 \mathrm{~m}$ and $\nu=0.3 \mathrm{~m}^{2} / \mathrm{s}$ at $74.25 \mathrm{~km}$, we find $\epsilon \approx 5 \times 10^{-5} \mathrm{~W} / \mathrm{kg}$, which is a factor $10^{3}$ lower than the lower limit on the dissipation rate in the turbulent PMWE layer just below (see Table 2).

\section{Conclusions}

In both rocket flights we see a distinct difference between the ion density fluctuation spectra inside and outside of altitude ranges with PMWE. Inside the PMWE the spectra display a $k^{-5 / 3}$ wavenumber dependence over about two decades, indicating the inertial subrange of a turbulent cascade. Outside the PMWE altitude ranges we see little or no signs of a turbulent cascade, with a $\sim k^{-3}$ spectrum at small wavenumbers and a $\sim k^{-7}$ spectrum at higher wavenumbers. Other observations (spectral broadening of radar signals consistent with turbulent velocity fluctuations, adiabatic lapse rates caused by turbulent mixing, etc.) from the same rocket campaign support this conclusion (Lübken et al., 2006).

At the boundaries of the PMWE regions the small scale fluctuations, at $k \gtrsim 10^{-1} \mathrm{~m}^{-1}$, quickly disappear as we move away from the PMWE. Interpreting this as an increase in the inner scale, it means that the energy dissipation rate is quickly reduced as we move up/down at the upper/lower boundaries of the PMWE. Inside the PMWE layers the smallscale fluctuations are located in several narrow sublayers.

The spectra thus provide evidence for turbulent processes in PMWE, and the slope of the spectra are consistent with damping of gravity waves through a turbulent cascade. Limitations in the instrumentation prevented us from measuring fluctuations at the Bragg wavelength, where PMWE are generated. Hence we can only put a lower limit on the turbulent energy dissipation rate, with $\epsilon \gtrsim 3 \times 10^{-5} \mathrm{~W} / \mathrm{kg}$ for the lowest PMWE layers (around $55 \mathrm{~km}$ ) and $\epsilon \gtrsim 10^{-2} \mathrm{~W} / \mathrm{kg}$ for the upper layers $(72 \mathrm{~km})$. This limitation also means that we cannot unambiguously identify the turbulent spectra with PMWE, since we have not established that the fluctuations at the Bragg wavelength are sufficient to produce radar backscatter of the right magnitude. Clearly, new rocket measurements with increased sensitivity at high frequencies are required to resolve these issues.

The present results and the results by Lübken et al. (2006) do not make any statement about the occurrence or nonoccurrence of charged particles, and they are consistent with an explanation for PMWE that does not involve charged aerosols. We conclude that observational data suggest that PMWE are caused by turbulence. Also, the data support the conclusion that we have a connection in both directions between turbulence and PMWE: Given a large enough electron density (for example due to a solar proton event), turbulence gives rise to PMWE, and conversely, if we observe PMWE, turbulence is also present.

Acknowledgements. The authors would like to thank professor O. Havnes and the engineers S. Olsen and T. Tønnesen at the University of Troms $\varnothing$, Norway, for their help.

Edited by: P. Hartogh

\section{References}

Balsley, B. B., Ecklund, W. L., and Fritts, D. C.: VHF echoes from the high-latitude mesosphere and lower thermosphere: Observations and interpretations, J. Atmos. Sci., 40, 2451-2466, 1983.

Belova, E., Kirkwood, S., Ekeberg, J., Osepian, A., Häggström, I., Nilsson, H., and Rietveld, M.: The dynamical background of polar mesosphere winter echoes from simultaneous EISCAT and ESRAD observations, Ann. Geophys., 23, 1239-1247, 2005, http://www.ann-geophys.net/23/1239/2005/.

Bilitza, D.: International Reference Ionosphere 2000, Radio Sci., 36, 261-275, 2001.

Blix, T. A., Thrane, E. V., Fritts, D. C., von Zahn, U., Lübken, F.J., Hillert, W., Blood, S. P., Mitchell, J. D., Kokin, G. A., and Pakhomov, S. V.: Small-scale structure observed in situ during MAC/EPSILON, J. Atmos. Terr. Phys., 52, 835-854, 1990a.

Blix, T. A., Thrane, E. V., and Andreassen, Ø.: In Situ Measurements of the Fine-Scale Structure and Turbulence in the Mesosphere and Lower Thermosphere by Means of Electrostatic Positive Ion Probes, J. Geophys. Res., 95, 5533-5548, 1990b. 
Blix, T. A., Thrane, E. V., Kirkwood, S., Dimant, Y. S., and Sudan, R. N.: Experimental evidence for unstable waves in the lower $E$ /upper $D$-region excited near the bisector between the electric field and the drift velocity, Geophys. Res. Lett., 23, 2137-2140, 1996.

Czechowsky, P., Rüster, R., and Schmidt, G.: Variations of mesospheric structures in different seasons, Geophys. Res. Lett., 6, 459-462, 1979.

Dimant, Y. S. and Sudan, R. N.: Kinetic theory of the FarleyBuneman instability in the $E$ region of the ionosphere, J. Geophys. Res., 100, 14605-14 623, 1995.

Ecklund, W. L. and Balsley, B. B.: Long-term observations of the Arctic mesosphere with the MST radar at Poker Flat, Alaska, J. Geophys. Res., 86, 7775-7780, 1981.

Folkestad, K.: Ionospheric studies by in situ measurements in sounding rockets, NDRE Report No. 59, Norwegian Defence Research Establishment, 1970.

Foufoula-Georgiou, E. and Kumar, P. (Eds.): Wavelets in Geophysics, Vol. 4 of Wavelet analysis and its applications, Academic Press, ISBN 0-12-262850-0, 1994.

Hunten, D. M., Turco, R. P., and Toon, O. B.: Smoke and dust particles of meteoric origin in the mesosphere and stratosphere, J. Atmos. Sci., 37, 1342-1357, 1980.

Jacobsen, T. A. and Friedrich, M.: Electron density measurements in the lower D-region, J. Atmos. Terr. Phys., 41, 1195-1200, 1979.

Kirkwood, S., Barabash, V., Belova, E., Nilsson, H., Rao, T. N., Stebel, K., Osepian, A., and Chilson, P. B.: Polar Mesosphere Winter Echoes during Solar Proton Events, Advances in Polar Upper Atmosphere Research, 16, 111-125, 2002.

Lie-Svendsen, Ø., Blix, T. A., and Hoppe, U.-P.: Modeling the plasma response to small-scale aerosol particle perturbations in the mesopause region, J. Geophys. Res., 108, 8442, doi:10.1029/2002JD002753, 2003.

Lübken, F.-J. and von Zahn, U.: Thermal Structure of the Mesopause Region at Polar Latitudes, J. Geophys. Res., 96, 20 841-20 857, 1991.

Lübken, F.-J., Hillert, W., Lehmacher, G., and von Zahn, U.: Experiments Revealing Small Impact of Turbulence on the Energy Budget of the Mesosphere and Lower Thermosphere, J. Geophys. Res., 98, 20 369-20384, 1993.
Lübken, F.-J., Strelnikov, B., Rapp, M., Singer, W., Latteck, R., Brattli, A., Hoppe, U.-P., and Friedrich, M.: The thermal and dynamical state of the atmosphere during polar mesosphere winter echoes, Atmos. Chem. Phys., 6, 13-24, 2006, http://www.atmos-chem-phys.net/6/13/2006/.

Lynch, K. A., Gelinas, L. J., Kelley, M. C., Collins, R. L., Widholm, M., Rau, D., MacDonald, E., Liu, Y., Ulwick, J., and Mace, P.: Multiple sounding rocket observations of charged dust in the polar winter mesosphere, J. Geophys. Res., 110, A03302, doi:10.1029/2004JA010502, 2005.

Mallat, S.: A wavelet tour of signal processing, Academic Press, 2nd edn., ISBN 0-12-466606-X, 1999.

Mechtly, A. A.: Accuracy of Rocket Measurements of Lower Ionosphere Electron Density Concentrations, Radio Sci., 9, 373-378, 1974.

Rapp, M. and Lübken, F.-J.: Polar mesosphere summer echoes (PMSE): review of observations and current understanding, Atmos. Chem. Phys., 4, 2601-2633, 2004, http://www.atmos-chem-phys.net/4/2601/2004/.

Rapp, M., Hedin, J., Strelnikova, I., Friedrich, M., Gumbel, J., and Lübken, F.-J.: Observations of positively charged nanoparticles in the nighttime polar mesosphere, Geophys. Res. Lett., 32, L23821, doi:10.1029/2005GL024676, 2005.

Sagalyn, R. C., Smiddy, M., and Wisnia, J.: Measurement and Interpretation of Ion Density Distributions in the Daytime $F$ Region, J. Geophys. Res., 68, 199-211, 1963.

Stebel, K., Blum, U., Fricke, K.-H., Kirkwood, S., Mitchell, N. J., and Osepian, A.: Joint radar/lidar observations of possible aerosol layers in the winter mesosphere, J. Atmos. Solar-Terr. Phys., 66, 957-970, 2004.

Strelnikov, B., Rapp, M., and Lübken, F.-J.: A new technique for the analysis of neutral air density fluctuations measured in situ in the middle atmosphere, Geophys. Res. Lett., 30, 2052, doi:10.1029/2003GL018271, 2003.

Torrence, C. and Compo, G. P.: A Practical Guide to Wavelet Analysis, Bull. Amer. Meteor. Soc., 79, 69-78, 1998.

Zeller, O., Zecha, M., Bremer, J., Latteck, R., and Singer, W.: Mean characteristics of mesosphere winter echoes at mid and high latitudes, J. Atmos. Solar-Terr. Phys., 68, 1087-1104, doi:10.1016/j.jastp.2006.02.015, 2006. 